\title{
ON AN INTEGRAL FORMULA OF GAUSS-BONNET-GROTEMEYER
}

\section{BANG-YEN CHEN}

ABstract. Let $\boldsymbol{e}(p)$ and $G(p)$ be the unit outer normal and the Gauss-Kronecker curvature of an oriented closed even-dimensional hypersurface $M$ of dimension $n$ in $E^{n+1}$. Then for a fixed unit vector $c$ in $E^{n+1}$, we have

$$
\begin{aligned}
\int_{M}(\mathbf{c} \cdot e)^{m} G d V & =c_{n+m} \chi(M) / c_{m}, & & \text { for } m=0,2,4, \cdots, \\
& =0, & & \text { for } m=1,3,5, \cdots,
\end{aligned}
$$

where $\boldsymbol{c} \cdot \boldsymbol{e}$ denotes the inner product of $\boldsymbol{c}$ and $\boldsymbol{e}, c_{m}$ the area of $m$ dimensional unit sphere, and $\chi(M)$ the Euler characteristic of $M$.

Let $M$ be an orientable closed hypersurface imbedded in a euclidean space $E^{n+1}$ of dimension $n+1 \geqq 3$. Let $\mathbf{x}(p)$ be the position vector of a point $p$ with respect to a fixed point 0 in $E^{n+1}$, and $e(p)$, $G(p)$ and $d V$ the unit outer normal, the Gauss-Kronecker curvature at $p$, and the volume element of $M$ in $E^{n+1}$, respectively. The main results of this paper are the following:

THEOREM 1. Let $M$ be an oriented closed hypersurface of dimension $n$ imbedded in euclidean space $E^{n+1}$ of dimension $n+1 \geqq 3$. Then we have

$$
\begin{aligned}
m \int_{M}(\mathbf{x} \cdot \mathbf{e})^{m-1} \mathbf{x} G d V=(n+m) \int_{M}(\mathbf{x} \cdot \mathbf{e})^{m} \mathrm{e} G d V & \\
& \quad m=0,1,2,3, \cdots .
\end{aligned}
$$

THEOREM 2. Under the same hypothesis of Theorem 1, if the dimension of $M$ is even, then for a fixed unit vector $\mathrm{c}$ in $E^{n+1}$, we have

$$
\begin{aligned}
\int_{M}(c \cdot e)^{m} G d V & =c_{n+m} \chi(M) / c_{m}, & & \text { for } m=0,2,4, \cdots, \\
& =0, & & \text { for } m=1,3,5, \cdots .
\end{aligned}
$$

REMARK. If $m=0$, then formula (3) is the well-known GaussBonnet formula, and if $m=2$ and $n=2$, then formula (3) was proved by Grotemeyer [3] in 1963.

Received by the editors May 22, 1970.

AMS 1969 subiect classifications. Primary 5340, 5374; Secondary 5372.

Key words and phrases. Closed hypersurface, differential form, Gauss-Kronecker curvature, Euler characteristic, Gauss-Bonnet-Grotemeyer formula. 
1. Preliminaries. Let $M$ be an oriented (differentiable) manifold of dimension $n$, and let $x: M \rightarrow E^{n+1}$ be a hypersurface. Let $e(p)$, $p \in M$, be the unit outer normal at $x(p)$. We consider the orthonormal frames $e_{1}, \cdots, e_{n}$ in the tangent hyperplane at $x(p)$, such that the determinant $\left(e_{1}, \cdots, e_{n}, e\right)=+1$. The space of all $e_{1}, \cdots, e_{n}$ can be identified with the principal fibre bundle $B$ of $M$ relative to the induced metric $d x \cdot d x$ (for the details, see Chern [2]). We have

$$
d x=\omega_{1} \mathbf{e}_{1}+\cdots+\omega_{n} \mathbf{e}_{n}, \quad d e=\theta_{1} \mathbf{e}_{1}+\cdots+\theta_{n} \mathbf{e}_{n},
$$

so that $\omega_{i}, \theta_{i}, 1 \leqq i \leqq n$, are linear differential forms in $B$. Since

$$
\mathbf{e} \cdot d x=0,
$$

we get, by exterior differentiation,

$$
d e \wedge d x=0 .
$$

The left-hand side in (6) is the exterior product of two vector-valued linear differential forms; vectors are multiplied in the sense of scalar products in $E^{n+1}$. In view of (4), equation (6) can be written

$$
\sum_{i} \omega_{i} \wedge \theta_{i}=0
$$

Since $\omega_{i}$ are linear independent, we can put, in view of (7),

$$
\theta_{i}=\sum A_{i j} \omega_{j}, \quad A_{i j}=A_{j i}, \quad 1 \leqq i, j \leqq n .
$$

The Gauss-Kronecker curvature $G$ is given by

$$
G=\operatorname{det}\left(A_{i j}\right) .
$$

Since $e_{1}, \cdots, e_{n}$ is an orthonormal frame, we know that the volume element $d V=\omega_{1} \wedge \cdots \wedge \omega_{n}$. Hence, by (8) and (9), we have

$$
\theta_{1} \wedge \cdots \wedge \theta_{n}=G d V \text {. }
$$

For simplicity, let $[, \ldots$,$] ( n$ terms) denote the combining operation of the vector product of $E^{n+1}$ with the exterior product. From (10), we have

$$
\begin{gathered}
(n \text { times }) \\
{[d e, \cdots, d e]=(n ! G d V) e .}
\end{gathered}
$$

2. Proof of Theorem 1. Put

$$
\delta=\sum_{i}(-1)^{i-1} \theta_{1} \wedge \cdots \wedge \hat{\theta}_{i} \wedge \cdots \wedge \theta_{n} e_{i},
$$

where "^" denotes the omitted term. Then, from (4), we have 


$$
\begin{array}{ll} 
& (n-1 \text { times }) \quad(n-1 \text { times }) \\
& {[d e, \cdots, d e, e]=\left[\sum \theta_{i} e_{i}, \cdots, \sum \theta_{i} e_{i}, \Theta\right]} \\
(13) \quad=(n-1) ! \sum \theta_{1} \wedge \cdots \wedge \hat{\theta}_{i} \wedge \cdots \wedge \theta_{n}\left[\theta_{1}, \cdots, \hat{e}_{i}, \cdots, \theta_{n}, \Theta\right] \\
=(n-1) ! \sum(-1)^{n-i-1} \theta_{1} \wedge \cdots \wedge \hat{\theta}_{i} \wedge \cdots \wedge \theta_{n} \theta_{i} \\
=(n-1) !(-1)^{n} \boldsymbol{d} .
\end{array}
$$

From (11) and (12), we get

$$
(n \text { times) }
$$

$$
d \mathbf{\sigma}=-[d e, \cdots, d e] /(n-1) !=-(n G d V) e .
$$

By (4), (12) and (14), we have

$$
\begin{aligned}
d\left((\mathbf{x} \cdot \mathbf{e})^{m} \mathbf{d}\right) & =m(\mathbf{x} \cdot \boldsymbol{e})^{m-1}(\mathbf{x} \cdot d \mathbf{e}) \wedge \boldsymbol{\delta}+(\mathbf{x} \cdot \mathbf{e})^{m} d \mathbf{\delta} \\
& =m(\mathbf{x} \cdot \boldsymbol{e})^{m-1} \sum\left(\mathbf{x} \cdot \mathbf{e}_{i}\right) \mathbf{e}_{i} \theta_{1} \wedge \cdots \wedge \theta_{n}+(\mathbf{x} \cdot \mathbf{e})^{m} d \mathbf{\delta} \\
& =m(\mathbf{x} \cdot \boldsymbol{e})^{m-1} \mathbf{x} G d V-(n+m)(\mathbf{x} \cdot \boldsymbol{e})^{m} e G d V .
\end{aligned}
$$

Integrating both sides of (15) over $M$ and applying Stokes' theorem, we get (2). This completes the proof of the theorem.

3. Proof of Theorem 2. Let $c$ be a unit vector in $E^{n+1}$. Taking the scalar product of $\mathbf{c}$ with both sides of (2), we get

$$
m \int_{M}(\mathbf{x} \cdot \mathbf{e})^{m-1}(\mathrm{x} \cdot \mathrm{c}) G d V=(n+m) \int_{M}(\mathbf{x} \cdot \mathbf{e})^{m}(\mathbf{c} \cdot \mathbf{e}) G d V
$$

We make the translation $x \rightarrow x+c$ of $M$. Then, by $\left(\mathrm{A}_{0}\right)$, we get

$$
\begin{aligned}
m \int_{M} \sum_{i_{1}=0}^{m-1}\left(\begin{array}{c}
m-1 \\
i_{1}
\end{array}\right) & (\mathrm{x} \cdot \mathrm{e})^{i_{1}}(\mathrm{c} \cdot \mathrm{e})^{m-i_{1}-1}((\mathrm{x} \cdot \mathrm{c})+1) G d V \\
& =(n+m) \int_{M} \sum_{i_{1}=0}^{m}\left(\begin{array}{c}
m \\
i_{1}
\end{array}\right)(\mathrm{x} \cdot \mathrm{e})^{i_{1}}(\mathrm{c} \cdot \mathrm{e})^{m-i_{1}+1} G d V .
\end{aligned}
$$

$\left(\mathrm{A}_{0}^{\prime}\right)-\left(\mathrm{A}_{0}\right)$ gives

$$
m \int_{M} \sum_{i_{1}=0}^{m-2}\left(\begin{array}{c}
m-1 \\
i_{1}
\end{array}\right)(\mathrm{x} \cdot \mathrm{e})^{i_{1}}(\mathrm{x} \cdot \mathrm{c})(\mathrm{c} \cdot \mathrm{e})^{m-i_{1}-1} G d V
$$

$$
\begin{aligned}
& +m \int_{M} \sum_{i_{1}=0}^{m-1}\left(\begin{array}{c}
m-1 \\
i_{1}
\end{array}\right)(\mathrm{x} \cdot e)^{i_{1}}(\mathrm{c} \cdot e)^{m-i_{1}-1} G d V \\
= & (n+m) \int_{M} \sum_{i_{1}=0}^{m-1}\left(\begin{array}{c}
m \\
i_{1}
\end{array}\right)(\mathrm{x} \cdot e)^{i_{1}}(\mathrm{c} \cdot e)^{m-i_{1}+1} G d V .
\end{aligned}
$$

Again we make the translation $x \rightarrow x+c$ of $M$ into $\left(A_{1}\right)$ and then subtract from $\left(A_{1}\right)$, we get 


$$
\begin{aligned}
& m \int_{M} \sum_{i_{1}=0}^{m-2}\left(\begin{array}{c}
m-1 \\
i_{1}
\end{array}\right) \sum_{i_{2}=0}^{i_{1}-1}\left(\begin{array}{c}
i_{1} \\
i_{2}
\end{array}\right)(\mathrm{x} \cdot \theta)^{i_{2}}(\mathrm{x} \cdot \mathrm{c})(\mathrm{c} \cdot \theta)^{m-i_{2}-1} G d V \\
& +m \int_{M}\left[\sum_{i_{1}=0}^{m-2}\left(\begin{array}{c}
m-1 \\
i_{1}
\end{array}\right) \sum_{i_{2}=0}^{i_{1}}\left(\begin{array}{c}
i_{1} \\
i_{2}
\end{array}\right)+\sum_{i_{1}=0}^{m-1}\left(\begin{array}{c}
m-1 \\
i_{1}
\end{array}\right) \sum_{i_{2}=0}^{i_{1}-1}\left(\begin{array}{l}
i_{1} \\
i_{2}
\end{array}\right)\right] \\
& \left(\mathrm{A}_{2}\right) \quad \cdot(\mathrm{x} \cdot \theta)^{i_{2}}(\mathrm{c} \cdot \theta)^{m-i_{2}-1} G d V \\
& =(n+m) \int \sum_{M}^{m-1}\left(\begin{array}{c}
m \\
i_{1}=0
\end{array}\right) \sum_{i_{2}=0}^{i_{1}-1}\left(\begin{array}{c}
i_{1} \\
i_{2}
\end{array}\right)(\mathrm{x} \cdot \theta)^{i_{2}}(\mathrm{c} \cdot \theta)^{m-i_{2}+1} G d V .
\end{aligned}
$$

Continuing this process $k$ times $(k=1,2, \cdots, m)$, we get

$$
\begin{aligned}
& m \int_{M} \sum_{i_{1}=0}^{m-2}\left(\begin{array}{c}
m-1 \\
i_{1}
\end{array}\right) \sum_{i_{2}=0}^{i_{1}-1}\left(\begin{array}{c}
i_{1} \\
i_{2}
\end{array}\right) \cdots \sum_{i_{k}=0}^{i_{k}-1-1}\left(\begin{array}{c}
i_{k-1} \\
i_{k}
\end{array}\right) \\
& \cdot(x \cdot e)^{i k}(x \cdot e)(c \cdot e)^{m-i k-1} G d V \\
& +m \int_{M}\left[\sum_{i_{1}=0}^{m-2}\left(\begin{array}{c}
m-1 \\
i_{1}
\end{array}\right) \sum_{i_{2}=0}^{i_{1}-1}\left(\begin{array}{l}
i_{1} \\
i_{2}
\end{array}\right)\right. \\
& \cdots \sum_{i_{k-1}=0}^{i_{k-2}-1}\left(\begin{array}{c}
i_{k-2} \\
i_{k-1}
\end{array}\right) \sum_{i_{k}=0}^{i_{k-1}}\left(\begin{array}{c}
i_{k-1} \\
i_{k}
\end{array}\right)+\cdots \\
& +\sum_{i_{1}=0}^{m-2}\left(\begin{array}{c}
m-1 \\
i_{1}
\end{array}\right) \sum_{i_{2}=0}^{i_{1}-1}\left(\begin{array}{c}
i_{1} \\
i_{2}
\end{array}\right) \cdots \sum_{i_{j}=0}^{i_{j-1}}\left(\begin{array}{c}
i_{j-1} \\
i_{j}
\end{array}\right) \\
& \ldots \sum_{i_{k}=0}^{i k-1-1}\left(\begin{array}{c}
i_{k-1} \\
i_{k}
\end{array}\right)+\cdots \\
& \left.+\sum_{i_{1}=0}^{m-1}\left(\begin{array}{c}
m-1 \\
i_{1}
\end{array}\right) \sum_{i_{2}=0}^{i_{1}-1}\left(\begin{array}{c}
i_{1} \\
i_{2}
\end{array}\right) \cdots \sum_{i_{k}=0}^{i_{k}-1-1}\left(\begin{array}{c}
i_{k-1} \\
i_{k}
\end{array}\right)\right] \\
& \cdot(\mathrm{x} \cdot \mathrm{e})^{i k}(\mathrm{c} \cdot \mathrm{e})^{m-i k-1} \mathrm{GdV} \\
& =(n+m) \int \sum_{M}^{m-1}\left(\begin{array}{l}
m \\
i_{1}
\end{array}\right) \sum_{i_{2}=0}^{i_{1}-1}\left(\begin{array}{l}
i_{1} \\
i_{2}
\end{array}\right) \cdots \sum_{i_{k}=0}^{i_{k}-1-1}\left(\begin{array}{c}
i_{k-1} \\
i_{k}
\end{array}\right) \\
& \cdot(\mathrm{x} \cdot \mathrm{e})^{i k}(\mathrm{c} \cdot \boldsymbol{\theta})^{m-i k+1} G d V .
\end{aligned}
$$

In particular, if $k=m$, then the first integral of $\left(\mathrm{A}_{k}\right)$ does not appear, and the terms in $[*+\cdots+*]$ in the second integrand is equal to $m$ !. Thus, $\left(A_{m}\right)$ gives us the following formula:

$$
\begin{aligned}
m \int_{M} \Psi(c \cdot e)^{m-1} G d V=(n+m) \int_{M}(c \cdot e)^{m+1} G d V & \\
& m=1,2,3, \ldots .
\end{aligned}
$$


Hence, we get

$$
\int_{M}(c \cdot e)^{m} G d V=\frac{m-1}{n+m-1} \int_{M}(c \cdot e)^{m-2} G d V .
$$

By the assumption, $n$ is even. Hence if $m$ is a positive even integer, then by (17), the Gauss-Bonnet formula and the fact

$$
c_{N}=2\left[\Gamma\left(\frac{1}{2}\right)\right]^{N+1} / \Gamma\left(\frac{1}{2}(N+1)\right),
$$

we get

$$
\begin{aligned}
\int_{M}(c \cdot e)^{m} G d V & =\frac{(m-1)(m-3) \cdots 1}{(n+m-1)(n+m-3) \cdots(n+1)} \int_{M} G d V \\
& =c_{n+m} \chi(M) / c_{m} .
\end{aligned}
$$

Moreover, by (2), we get

$$
\int_{M} e G d V=0 .
$$

Taking the inner product of $c$ with (20), we get

$$
\int_{M}(c \cdot e) G d V=0 .
$$

Hence, in view of (16) and (21), we find that

$$
\int_{M}(c \cdot e)^{m} G d V=0, \quad \text { for all } m=1,3,5, \cdots \text {. }
$$

Therefore, by (19), (22) and the Gauss-Bonnet formula, we get formula (3). This completes the proof of the theorem.

The author would like to express his hearty thanks to Professor T. Nagano for valuable conversations about this paper.

\section{REFERENCES}

1. B.-Y. Chen, On the total curvature of immersed manifolds. I: An inequality of Fenchel-Borsuk-Willmore, Amer. J. Math. (to appear).

2. S.-S. Chern, Integral formulas for hypersurfaces in Euclidean space and their applications to uniqueness theorems, J. Math. Mech. 8 (1959), 947-955. MR 22 \#4997.

3. K. P. Grotemeyer, Über das Normalenbindel differenzierbarer Mannig faltigkeiten, Ann. Acad. Sci. Fenn. Ser. A. I. No. 336/15 (1963). MR 29 \#568.

University of Notre Dame, Notre Dame, Indiana 46556

Michigan State University, East Lansing, Michigan 48823 